\title{
Efficient Synthesis of $\mathbf{N}^{\alpha}, \mathbf{N}$-Disubstituted $\alpha$-Aminocarbohydroxamic Acids
}

\author{
Detlef Geffken*, Alf Ploetz and Hans v. Zydowitz
}

Department of Pharmaceutical Chemistry, University of Hamburg, Bundesstrasse 45, 20146 Hamburg, Germany

The title compounds 3 can be prepared in good yields by the reaction of $\mathrm{N}$-substituted $\alpha$-chlorocarbohydroxamic acids 2 and primary amines in dimethylacetamide as solvent.

Keywords: N-substituted a-chlorocarbohydroxamic acids, primary amines, dimethylacetamide, $\mathrm{N}^{\alpha}, \mathrm{N}$-disubstituted $\alpha$-aminocarbohydroxamic acids

\section{Introduction}

$\mathrm{N}$-Unsubstituted $\alpha$-aminocarbohydroxamic acids have attained growing interest in medicinal chemistry as building blocks for enzyme inhibitors [1,2]. Surprisingly, only little work has been devoted to $\mathrm{N}^{\alpha}, \mathrm{N}$-disubstituted $\alpha$-aminocarbohydroxamic acids until now. Meier and Boche [3] investigated some $\mathrm{N}$-aryl- $\mathrm{N}^{\alpha}$-benzylaminocarbohydroxamic acids and their rearrangement to the corresponding $\mathrm{O}$-acyl derivatives which are suspected to be ultimate carcinogens of aromatic amines [4] and Ono and Itoh [5] described $\mathrm{N}^{\alpha}$-dimethylamino-Nmethylacetohydroxamic acid as a reagent for selective cleavage of carboxylic esters under neutral conditions.

As a part of our research directed to bioactive molecules derived from bifunctional hydroxamic acids we needed a series of $\mathrm{N}^{\alpha}, \mathrm{N}$-disubstituted $\alpha$-aminocarbohydroxamic acids 3 and found the target compounds to be readily available by reacting $\alpha$-chlorocarbohydroxamic acids 2 with primary amines (Scheme 1). 
Scheme 1<smiles>[R]NC([R])C(=O)N([R])O</smiles>

i: $\mathbf{R}^{2} \mathrm{NHOH}, \mathrm{NaHCO}_{3} ;$ ii: $\mathbf{R}^{3} \mathrm{NH}_{2}$, dimethylacetamide, $25^{\circ} \mathrm{C} ; \mathbf{1 a}: \mathbf{R}^{\mathbf{1}}=\mathrm{H}, \mathbf{1 b}: \mathbf{R}^{\mathbf{1}}=\mathbf{M e} ; \mathbf{1 c}: \mathbf{R}^{1}=\mathbf{P h}$ 2a: $\mathbf{R}^{1}=\mathrm{H}, \mathrm{R}^{2}=\mathrm{CH}_{2} \mathrm{Ph} ; \mathbf{2 b}: \mathbf{R}^{1}=\mathrm{H}, \mathbf{R}^{2}=\mathrm{CH}(\mathrm{Ph})_{2} ; \mathbf{2 c}: \mathrm{R}^{1}=\mathrm{H}, \mathbf{R}^{2}=\mathrm{i}$-prop; $2 \mathrm{~d}: \mathbf{R}^{1}=\mathrm{H}, \mathrm{R}^{2}=\mathrm{CH}_{2}(2,4 \mathrm{Cl}) \mathrm{Ph}$; 2e: $\mathrm{R}^{1}=\mathrm{CH}_{3}, \mathrm{R}^{2}=\mathrm{CH}(\mathrm{Ph})_{2} ; 2 \mathrm{ff}: \mathbf{R}^{1}=\mathrm{Ph}, \mathrm{R}^{2}=\mathrm{CH}_{3} ; 2 \mathrm{~g}: \mathrm{R}^{1}=\mathrm{Ph}, \mathrm{R}^{2}=\mathrm{CH}_{2} \mathrm{Ph} ; \mathbf{2 h}: \mathrm{R}^{1}=\mathrm{Ph}, \mathrm{R}^{2}=2 \mathrm{~F}-\mathrm{Ph}$; 2i: $\mathrm{R}^{1}=\mathrm{H}, \mathrm{R}^{2}=\mathrm{CH}_{3}$

\section{Results and Discussion}

$\alpha$-Chlorocarbohydroxamic acids 2 could easily be prepared according to Exner [6] in yields of $55-85 \%$ by dropping a solution of $\alpha$-chlorocarboxylic acid chloride in diethyl ether into a heavily stirred two phase system of diethyl ether/water containing the appropriate Nsubstituted hydroxylamine.

Table 1:

\begin{tabular}{|c|c|c|c|}
\hline 3 & $\mathbf{R}^{1}$ & $\mathrm{R}^{2}$ & $\mathbf{R}^{3}$ \\
\hline a & $\mathrm{H}$ & $\mathrm{Me}$ & $\mathrm{Ph}$ \\
\hline b & $\mathrm{H}$ & $\mathrm{Me}$ & $\mathrm{CH}_{2}(4-\mathrm{Cl}) \mathrm{Ph}$ \\
\hline c & $\mathrm{H}$ & $\mathrm{Me}$ & $\mathrm{CH}_{2}(4-\mathrm{MeO}) \mathrm{Ph}$ \\
\hline d & $\mathrm{H}$ & $\mathrm{Me}$ & $\left(\mathrm{CH}_{2}\right)_{2} \mathrm{Ph}$ \\
\hline e & $\mathrm{H}$ & $\mathrm{CH}(\mathrm{Ph})_{2}$ & $\mathrm{Ph}$ \\
\hline$f$ & $\mathrm{H}$ & $\mathrm{CH}_{2} \mathrm{Ph}$ & $\mathrm{Ph}$ \\
\hline $\mathrm{g}$ & $\mathrm{H}$ & $\mathrm{CH}(\mathrm{Ph})_{2}$ & $\mathrm{CH}_{2} \mathrm{Ph}$ \\
\hline $\mathrm{h}$ & $\mathbf{H}$ & $\mathrm{CH}_{2}(2,4-\mathrm{Cl}) \mathrm{Ph}$ & $\mathrm{CH}_{2} \mathrm{Ph}$ \\
\hline $\mathrm{i}$ & $\mathrm{H}$ & i-prop & $\mathrm{Ph}$ \\
\hline$j$ & $\mathrm{Me}$ & $\mathrm{Me}$ & $\mathrm{CH}_{2} \mathrm{Ph}$ \\
\hline $\mathrm{k}$ & $\mathrm{Ph}$ & $\mathrm{Me}$ & $\mathrm{Ph}$ \\
\hline 1 & $\mathrm{Ph}$ & $\mathrm{CH}_{2} \mathrm{Ph}$ & $\mathrm{CH}_{2} \mathrm{Ph}$ \\
\hline $\mathrm{m}$ & $\mathrm{Ph}$ & $2 \mathrm{~F}-\mathrm{Ph}$ & $\mathrm{CH}_{2} \mathrm{Ph}$ \\
\hline $\mathrm{n}$ & $\mathrm{Ph}$ & $2 \mathrm{~F}-\mathrm{Ph}$ & $\mathrm{Ph}$ \\
\hline o & $\mathrm{H}$ & $\mathrm{CH}_{2} \mathrm{Ph}$ & $\mathrm{Ph}$ \\
\hline
\end{tabular}

Initial aminolysis studies of $\mathbf{2}$ indicated a remarkable influence of the solvent on the formation of 3: Yields were unsatisfactory when running the experiments in diethyl ether, ethyl acetate or acetonitrile. However, good results were obtained by reacting 2 in dimethylacetamide with a primary amine in a molar ratio of 1:3 at ambient temperature for 12-24h. Simply work-up of the reaction mixture by diluting with ice-cold water provided 3a-o (Table 1) in 53-88\% yields 
as crystalline compounds, characterized by elemental analysis, IR and ${ }^{1} \mathrm{H}-\mathrm{NMR}$ spectra (see experimental part) and a typical purple color reaction with ferric chloride in ethanol.

In conclusion, a simple synthetic approach to $\mathrm{N}^{\alpha}, \mathrm{N}$-substituted $\alpha$-aminocarbohydroxamic acids 3 has been developed starting from commercially available $\alpha$-chlorocarboxylic acid chlorides. Heterocyclizations of 3, providing novel ring systems as well as biological activities of $\mathbf{3}$ and derivatives thereof will be reported in due course.

\section{Experimental Part}

Melting points (uncorrected) were taken in open capillary tubes using a Mettler FP 62 apparatus. 'H-NMR spectra were recorded on a Bruker AMX 400 spectrometer with TMS as the internal standard. The IR spectra $(\mathrm{KBr})$ were recorded on a ATI Mattson Genesis Series FTIR. Elemental analyses were performed on a Heraeus CHN-O-Rapid. For all compounds satisfactory microanalyses were obtained.

General Procedure for the Preparation of $\mathrm{N}$-substituted $\alpha$-chlorocarbohydroxamic acids 2a-i:

To a solution of sodium carbonate $(10 \mathrm{mmol})$ in $10 \mathrm{ml} \mathrm{H} \mathrm{H}_{2} \mathrm{O}$ was slowly added the corresponding $\mathrm{N}$-substituted hydroxylamine $(10 \mathrm{mmol})$ hydrochloride. After addition of 60 $\mathrm{ml}$ diethyl ether the mixture was cooled in an ice bath and a solution of $\alpha$-chlorocarboxylic acid chloride $(10 \mathrm{mmol})$ in $10 \mathrm{ml}$ diethyl ether was added dropwise under vigorous stirring. After stirring the mixture at ambient temperature for $3 \mathrm{~h}$ the organic layer was separated and the aqueous solution extracted thrice with $10 \mathrm{ml}$ diethyl ether. The combined organic layers were dried $\left(\mathrm{MgSO}_{4}\right)$ and evaporated. Oily residues crystallized from diethyl ether/petrolether within $24 \mathrm{~h}$ on standing in the refrigerator. A single recrystallization of the crude products furnished pure $\mathbf{2 a - h}$.

\section{N-Benzyl- $\alpha$-chloroacetohydroxamic acid (2a)}

Yield 92\%; m.p $82^{\circ} \mathrm{C}$; IR: $3173(\mathrm{OH}), 1634 \mathrm{~cm}^{-1}\left(\mathrm{C}=\mathrm{O}\right.$ ); ${ }^{1} \mathrm{H}-\mathrm{NMR}$ (DMSO-d ) $_{6} \delta 4.45$ (s, $2 \mathrm{H}$ ), 4.71 (s, 2H), 7.27-7.37 (m, $5 \mathrm{Ar}-\mathrm{H}$ ), 10.17 (s, OH); $\mathrm{C}_{9} \mathrm{H}_{10} \mathrm{CNNO}_{2}$ (199.6).

$\boldsymbol{\alpha}$-Chloro- $\mathbf{N}$-(diphenylmethyl)acetohydroxamic acid (2b)

Yield 71\%; m.p $128^{\circ} \mathrm{C}$; IR: $3153(\mathrm{OH}), 1630 \mathrm{~cm}^{-1}(\mathrm{C}=0)$ ) ${ }^{1} \mathrm{H}-\mathrm{NMR}$ (DMSO-d 6 ): $\delta 4.52$ (s, $2 \mathrm{H}), 6.75(\mathrm{~s}, 1 \mathrm{H}), 7.20-7.40$ (m, $10 \mathrm{Ar}-\mathrm{H}), 9.90(\mathrm{~s}, \mathrm{OH}) ; \mathrm{C}_{13} \mathrm{H}_{14} \mathrm{ClNO}_{2}$ (275.7).

$\alpha$-Chloro- $\mathrm{N}$-ispropylacetohydroxamic acid (2c)

Yield 48\%; m.p $85^{\circ} \mathrm{C}$; IR: $3127(\mathrm{OH}), 1623 \mathrm{~cm}^{-1}(\mathrm{C}=0)$; ${ }^{1} \mathrm{H}-\mathrm{NMR}$ (DMSO-d $\left.\mathrm{d}_{6}\right): \delta 1.05$ (d, J $=7.1 \mathrm{~Hz}, 6 \mathrm{H}), 4.36(\mathrm{~s}, 2 \mathrm{H}), 4.50(\mathrm{sept}, \mathrm{J}=7.1 \mathrm{~Hz}, 1 \mathrm{H}), 9.64(\mathrm{~s}, \mathrm{OH}) ; \mathrm{C}_{5} \mathrm{H}_{10} \mathrm{ClNO}_{2}(151.6)$. 
$\alpha$-Chloro-N-(2,4-dichlorophenyl)acetohydroxamic acid (2d)

Yield 75\%; m.p $131^{\circ} \mathrm{C}$; IR: $3187(\mathrm{OH}), 1635 \mathrm{~cm}^{-1}(\mathrm{C}=\mathrm{O})$; ${ }^{1} \mathrm{H}-\mathrm{NMR}$ (DMSO-d $\mathrm{d}_{6}$ ): $\delta 4.49$ (s, $2 \mathrm{H}), 4.80(\mathrm{~s}, 2 \mathrm{H}), 7.36-7.65$ (m, $3 \mathrm{Ar}-\mathrm{H}), 10.25(\mathrm{~s}, \mathrm{OH}) ; \mathrm{C}_{9} \mathrm{H}_{8} \mathrm{ClNO}_{2}(268.5)$.

$\boldsymbol{\alpha}$-Chloro- $\mathrm{N}$-(diphenylmethyl)propiohydroxamic acid (2e)

Yield 58\%; m.p $130^{\circ} \mathrm{C}$; IR: $3210(\mathrm{OH}), 1633 \mathrm{~cm}^{-1}(\mathrm{C}=\mathrm{O})$; ${ }^{1} \mathrm{H}-\mathrm{NMR}$ (DMSO-d $\left.{ }_{6}\right): \delta 1.56(\mathrm{~d}, \mathrm{~J}$ $=6.6 \mathrm{~Hz}, 3 \mathrm{H}), 5.12(\mathrm{q}, \mathrm{J}=6.6 \mathrm{~Hz}, 1 \mathrm{H}), 6.72(\mathrm{~s}, 1 \mathrm{H}), 7.24-7.37(\mathrm{~m}, 10 \mathrm{Ar}-\mathrm{H}), 9.97(\mathrm{~s}, \mathrm{OH})$;

$\mathrm{C}_{16} \mathrm{H}_{16} \mathrm{CINO}_{2}$ (289.8).

$\alpha$-Chloro-N-methyl-phenylacetohydroxamic acid (2f)

Yield 78\%; m.p 96 $9{ }^{\circ} \mathrm{C}$; IR: $3185(\mathrm{OH}), 1638 \mathrm{~cm}^{-1}(\mathrm{C}=\mathrm{O})$; ${ }^{1} \mathrm{H}-\mathrm{NMR}$ (DMSO-d $\mathrm{d}_{6}$ ): $\delta 3.12$ (s, $3 \mathrm{H}), 6.20(\mathrm{~s}, 1 \mathrm{H})$, 7.37-7.50 (m, $5 \mathrm{Ar}-\mathrm{H}), 10.32(\mathrm{~s}, \mathrm{OH}) ; \mathrm{C}_{9} \mathrm{H}_{10} \mathrm{CINO}_{2}(199.6)$.

\section{N-Benzyl- $\alpha$-chlorophenylacetohydroxamic acid (2g)}

Yield 82\%; m.p $138^{\circ} \mathrm{C}$; IR: $3110(\mathrm{OH}), 1630 \mathrm{~cm}^{-1}(\mathrm{C}=\mathrm{O})$; ${ }^{1} \mathrm{H}-\mathrm{NMR}$ (DMSO-d $\left.\mathrm{d}_{6}\right): \delta 4.58$ (q $2 \mathrm{H}), 6.20(\mathrm{~s}, 1 \mathrm{H}), 7.15-7.52(\mathrm{~m}, 10 \mathrm{Ar}-\mathrm{H}), 10.25(\mathrm{~s}, \mathrm{OH}) ; \mathrm{C}_{15} \mathrm{H}_{14} \mathrm{ClNO}_{2}(275.7)$

$\alpha$-Chloro-N-(2-fluorophenyl)phenylacetohydroxamic acid (2h)

Yield 73\%; m.p $144^{\circ} \mathrm{C}$; IR: $3241(\mathrm{OH}), 1648 \mathrm{~cm}^{-1}(\mathrm{C}=\mathrm{O}) ;{ }^{1} \mathrm{H}-\mathrm{NMR}$ (DMSO-d $\mathrm{d}_{6}$ ): $\delta 6.40$ (s, 1H), 7.30-7.55 (m, $9 \mathrm{Ar}-\mathrm{H}), 11.13$ (s, OH); $\mathrm{C}_{14} \mathrm{H}_{11} \mathrm{Cl}_{\mathrm{F}} \mathrm{NO}_{2}$ (279.7)

$\boldsymbol{\alpha}$-Chloro-N-methyl-acetohydroxamic acid (2i) was prepared according to literature [6]

General Procedure for the Preparation of $\mathrm{N}^{\alpha}, \mathrm{N}$-substituted $\alpha$-aminocarbohydroxamic acids 3a-0:

To a solution of $2(10 \mathrm{mmol})$ in $20 \mathrm{ml}$ dimethylacetamide was added the appropriate amine ( $30 \mathrm{mmol}$ ), the mixture was stirred at ambient temperature for $48 \mathrm{~h}$ and finally poured on ice. The precipitate was separated and recrystallized from ethyl acetate furnishing pure 3a-o.

$\mathrm{N}$-Methyl- $\alpha$-(phenylamino)acetohydroxamic acid (3a)

From 2i [6] and aniline; yield 68\%; m.p $126^{\circ} \mathrm{C}$; IR: $\left.3386 \mathrm{NH}\right), 3236$ (br, OH), $1621 \mathrm{~cm}^{-1}$ $(\mathrm{C}=\mathrm{O})$; ${ }^{1} \mathrm{H}-\mathrm{NMR}\left(\mathrm{DMSO}_{\mathrm{d}}\right.$ ): $\delta 3.14$ (s, 3H), 3.92 (s, 2H), 6.53-7.08 (m, $\left.5 \mathrm{Ar}-\mathrm{H}\right), 9.99$ (s, $\mathrm{OH}) ; \mathrm{C}_{9} \mathrm{H}_{12} \mathrm{~N}_{2} \mathrm{O}_{2}$ (180.2).

$\alpha$-(4-Chlorobenzylamino)-N-methyl-acetohydroxamic acid (3b)

From 2i and 4-chlorobenzylamine; yield 88\%; m.p $166^{\circ} \mathrm{C}$; IR: $3271(\mathrm{NH}), 2461$ (br, $\left.\mathrm{OH}\right)$, $1637 \mathrm{~cm}^{-1}(\mathrm{C}=\mathrm{O})$; ${ }^{1} \mathrm{H}-\mathrm{NMR}$ (DMSO-d $\left.\mathrm{d}_{6}\right): \delta 3.09(\mathrm{~s}, 3 \mathrm{H}), 3.30(\mathrm{~s}, 2 \mathrm{H}), 3.68(\mathrm{~s}, 2 \mathrm{H}), 7.29-7.44$ (m, 4 Ar-H), 10.09 (s, OH); $\mathrm{C}_{10} \mathrm{H}_{13} \mathrm{ClN}_{2} \mathrm{O}_{2}$ (228.7).

$\alpha$-(4-Methoxybenzylamino)-N-methyl-acetohydroxamic acid (3c) 
From 2i and 4-methoxybenzylamine; yield 85\%; m.p 152 ${ }^{\circ} \mathrm{C}$; IR: $3262(\mathrm{NH}), 2485$ (br, OH), $1638 \mathrm{~cm}^{-1}(\mathrm{C}=\mathrm{O})$; ${ }^{1} \mathrm{H}-\mathrm{NMR}$ (DMSO-d $\left.\mathrm{d}_{6}\right): \delta 3.10(\mathrm{~s}, 3 \mathrm{H}), 3.30(\mathrm{~s}, 2 \mathrm{H}), 3,62(\mathrm{~s}, 2 \mathrm{H}), 6.88(\mathrm{~d}, 2$ Ar-H), 7.23 (d, 2H), 10.09 (s, OH); $\mathrm{C}_{11} \mathrm{H}_{16} \mathrm{~N}_{2} \mathrm{O}_{3}$ (224.3).

N-Methyl- $\alpha$-(2-phenylethylamino)acetohydroxamic acid (3d)

From 2i and 2-phenylethylamine; yield 64\%; m.p $136^{\circ} \mathrm{C}$; IR: 3300 (NH), 2448 (br, OH), $1644 \mathrm{~cm}^{-1}(\mathrm{C}=0)$ ) ' ${ }^{1} \mathrm{H}-\mathrm{NMR}$ (DMSO-d $\left.\mathrm{d}_{6}\right): \delta 2.60-2.80(\mathrm{~m}, 4 \mathrm{H}), 3.09(\mathrm{~s}, 3 \mathrm{H}), 3.41(\mathrm{~s}, 2 \mathrm{H})$, 7.17-7.29; (m, $5 \mathrm{Ar}-\mathrm{H}) ; \mathrm{C}_{11} \mathrm{H}_{16} \mathrm{~N}_{2} \mathrm{O}_{2}$ (208.3).

\section{$\mathbf{N}$-(Diphenylmethyl)- $\alpha$-(phenylamino)acetohydroxamic acid (3e)}

From 2b and aniline; yield 73\%; m.p $145^{\circ} \mathrm{C}$; IR: 3409 (NH), 3263 (br, OH), $1639 \mathrm{~cm}^{-1}$ $(\mathrm{C}=0)$ ) ${ }^{1} \mathrm{H}-\mathrm{NMR}\left(\mathrm{DMSO}_{\mathrm{d}}\right)$ ): $\delta 4.10(\mathrm{~s}, 2 \mathrm{H}), 3.91(\mathrm{~s}, 2 \mathrm{H}), 6.55(\mathrm{~m}, 3 \mathrm{Ar}-\mathrm{H}), 6.76(\mathrm{~s}, 1 \mathrm{H})$, 7.08 (m, 2Ar-H), 7.22-7.40 (m, $10 \mathrm{Ar}-\mathrm{H}), 9.80(\mathrm{~s}, \mathrm{OH}) ; \mathrm{C}_{21} \mathrm{H}_{20} \mathrm{~N}_{2} \mathrm{O}_{2}$ (332.4).

\section{$\alpha$-(Benzylamino)- $\mathrm{N}$-methyl-acetohydroxamic acid (3f)}

From 2i and benzylamine; yield 79\%; m.p $153^{\circ} \mathrm{C}$; IR: $3270(\mathrm{NH}), 2446(\mathrm{br}, \mathrm{OH}), 1644 \mathrm{~cm}^{-1}$ $(\mathrm{C}=\mathrm{O})$; ${ }^{1} \mathrm{H}-\mathrm{NMR}\left(\mathrm{DMSO}-\mathrm{d}_{6}\right): \delta 3.10(\mathrm{~s}, 3 \mathrm{H}), 3.35(\mathrm{~s}, 2 \mathrm{H}), 3.70(\mathrm{~s}, 2 \mathrm{H}), 7.15-7.30(\mathrm{~m}, 5 \mathrm{Ar}-$ $\mathrm{H}), 10.09$ (s, OH); $\mathrm{C}_{10} \mathrm{H}_{14} \mathrm{~N}_{2} \mathrm{O}_{2}$ (194.2).

\section{$\alpha$-(Benzylamino)-N-(diphenylmethyl)acetohydroxamic acid (3g)}

From 2b and benzylamine; yield 73\%; m.p $122^{\circ} \mathrm{C}$; IR: $3291(\mathrm{NH}), 2580(\mathrm{br}, \mathrm{OH}), 1655 \mathrm{~cm}^{-1}$ $(\mathrm{C}=0)$; ' $\mathrm{H}-\mathrm{NMR}\left(\mathrm{DMSO}-\mathrm{d}_{6}\right)$ : $\delta 3.57(\mathrm{~s}, 2 \mathrm{H}), 3.71(\mathrm{~s}, 2 \mathrm{H}), 6.78(\mathrm{~s}, 1 \mathrm{H}), 7.10-7.47$ (m, $15 \mathrm{Ar}-$ $\mathrm{H}), 9.60(\mathrm{~s}, \mathrm{OH}) ; \mathrm{C}_{22} \mathrm{H}_{22} \mathrm{~N}_{2} \mathrm{O}_{2}$ (346.4).

$\alpha$-(Benzylamino)-N-(2,4-dichlorobenzyl)acetohydroxamic acid (3h)

From 2d and 2,4-dichlorobenzylamine; yield 93\%; m.p $152^{\circ} \mathrm{C}$; IR: 3274 (NH), 2498 (br, $\mathrm{OH}), 1644 \mathrm{~cm}^{-1}(\mathrm{C}=\mathrm{O})$; ${ }^{1} \mathrm{H}-\mathrm{NMR}$ (DMSO-d $\left.\mathrm{d}_{6}\right): \delta 3.49$ (s, 2H), $3.72(\mathrm{~s}, 2 \mathrm{H}), 4.79(\mathrm{~s}, 2 \mathrm{H}), 7.18-$ $7.66(\mathrm{~m}, 8 \mathrm{Ar}-\mathrm{H}) ; \mathrm{C}_{16} \mathrm{H}_{16} \mathrm{Cl}_{2} \mathrm{~N}_{2} \mathrm{O}_{3}$ (339.2).

\section{$\mathrm{N}-$ Isopropyl- $\alpha$-(phenylamino)acetohydroxamic acid (3i)}

From 2c and aniline; yield 65\%; m.p $103^{\circ} \mathrm{C}$; IR: $3390(\mathrm{NH}), 3221(\mathrm{OH}), 1607 \mathrm{~cm}^{-1}(\mathrm{C}=\mathrm{O})$; ${ }^{1} \mathrm{H}-\mathrm{NMR}$ (DMSO-d $\mathrm{d}_{6}$ ): $\delta 1.10$ (d, J = 7.1 Hz, 6H), 3.90 (s, 2H), 4.55 (q, J = 7.1 Hz, 1H), 5.54 $(\mathrm{s}, 1 \mathrm{H}), 6.52-7.12(\mathrm{~m}, 5 \mathrm{Ar}-\mathrm{H}), 9.49(\mathrm{~s}, \mathrm{OH}) ; \mathrm{C}_{11} \mathrm{H}_{16} \mathrm{~N}_{2} \mathrm{O}_{2}(208.3)$.

$\alpha$-(Benzylamino)- $\mathrm{N}$-methyl-propiohydroxamic acid (3j)

From 2e and benzylamine; yield 53\%; m.p $121^{\circ} \mathrm{C}$; IR: $3268(\mathrm{NH}), 2447(\mathrm{br}, \mathrm{OH}), 1631 \mathrm{~cm}^{-1}$ $(\mathrm{C}=\mathrm{O})$; ${ }^{1} \mathrm{H}-\mathrm{NMR}$ (DMSO-d $)_{6}$ : $\delta 1.10(\mathrm{~d}, 3 \mathrm{H}), 3.13(\mathrm{~s}, 3 \mathrm{H}$, ), 3.55 (dd, 2H), 3.75 (q, 1H), 7.20 $7.37(\mathrm{~m}, 5 \mathrm{Ar}-\mathrm{H}), 9.88(\mathrm{~s}, \mathrm{OH}) ; \mathrm{C}_{11} \mathrm{H}_{16} \mathrm{~N}_{2} \mathrm{O}_{2}$ (208.3).

$\mathrm{N}$-Methyl- $\alpha$-(phenylamino)-phenylacetohydroxamic acid (3k) 
From $2 \mathrm{f}$ and aniline; yield 63\%; m.p $146^{\circ} \mathrm{C}$; IR: $3398(\mathrm{NH}), 3265(\mathrm{OH}), 1625 \mathrm{~cm}^{-1}(\mathrm{C}=\mathrm{O})$; ${ }^{1} \mathrm{H}-\mathrm{NMR}$ (DMSO-d $\mathrm{d}_{6}$ ): $\delta 3.11(\mathrm{~s}, 3 \mathrm{H}), 5.66(\mathrm{~d}, 1 \mathrm{H}), 6.03(\mathrm{~d}, 1 \mathrm{H}), 6.52-7.48(\mathrm{~m} 10 \mathrm{Ar}-\mathrm{H})$, 10.19 (s, OH); $\mathrm{C}_{15} \mathrm{H}_{16} \mathrm{~N}_{2} \mathrm{O}_{2}$ (256.3).

\section{N-Benzyl- $\alpha$-(benzylamino)phenylacetohydroxamic acid (31)}

From 2g and benzylamine; yield 63\%; m.p $102^{\circ} \mathrm{C}$; IR: $3260(\mathrm{NH}), 3040(\mathrm{br}, \mathrm{OH}), 1630 \mathrm{~cm}^{-1}$ $(\mathrm{C}=0)$ ) ${ }^{1} \mathrm{H}-\mathrm{NMR}\left(\mathrm{DMSO}-\mathrm{d}_{6}\right)$ : $\delta$ 3.62(d, 2H), $3.72(\mathrm{~d}, 2 \mathrm{H}), 4.90(\mathrm{~s}, 1 \mathrm{H}), 5.66(\mathrm{~d}, 1 \mathrm{H}), 6.03(\mathrm{~d}$, 1H), 7.10-7.40 (m $15 \mathrm{Ar}-\mathrm{H}), 9.50$ (s, OH); $\mathrm{C}_{22} \mathrm{H}_{22} \mathrm{~N}_{2} \mathrm{O}_{2}$ (346.4)

$\alpha$-(Benzylamino)-N-(2-fluorophenyl)phenylacetohydroxamic acid (3m)

From $2 \mathrm{~h}$ and benzylamine; yield 65\%; m.p $135^{\circ} \mathrm{C}$; IR: $3306(\mathrm{NH}), 3156(\mathrm{br}, \mathrm{OH}), 1627 \mathrm{~cm}^{-1}$ $(\mathrm{C}=\mathrm{O})$; ${ }^{1} \mathrm{H}-\mathrm{NMR}$ (DMSO-d $\left.\mathrm{d}_{6}\right): \delta 3.55$ (q, 2H), 5.06 (s, 1H), 7.18-7.48 (m $\left.9 \mathrm{Ar}-\mathrm{H}\right), 10.76$ (s, $\mathrm{OH}) ; \mathrm{C}_{21} \mathrm{H}_{19} \mathrm{FN}_{2} \mathrm{O}_{2}$ (350.4).

\section{$\mathbf{N}$-(2-Fluorophenyl)- $\alpha$-(phenylamino)phenylacetohydroxamic acid (3n )}

From $2 \mathrm{~h}$ and aniline; yield 55\%; m.p 141 ${ }^{\circ} \mathrm{C}$; IR: $3398(\mathrm{NH}), 3258(\mathrm{br}, \mathrm{OH}), 1632 \mathrm{~cm}^{-1}$ $(\mathrm{C}=\mathrm{O})$; ${ }^{1} \mathrm{H}-\mathrm{NMR}$ (DMSO-d $\mathrm{d}_{6}$ ): $\delta 3.55$ (q, 2H), 5.90 (s, 1H), 7.18-7.54 (m $\left.14 \mathrm{Ar}-\mathrm{H}\right), 11.00$ (s, $\mathrm{OH}) ; \mathrm{C}_{20} \mathrm{H}_{17} \mathrm{FN}_{2} \mathrm{O}_{2}$ (336.4).

\section{$\mathrm{N}$-Benzyl- $\alpha$-(phenylamino)acetohydroxamic acid (3o )}

From 2a and aniline; yield 73\%; m.p $141^{\circ} \mathrm{C}$; IR: $3273(\mathrm{NH}), 2780(\mathrm{br}, \mathrm{OH}), 1646 \mathrm{~cm}^{-1}$ $(\mathrm{C}=\mathrm{O})$; ${ }^{1} \mathrm{H}-\mathrm{NMR}\left(\mathrm{DMSO}-\mathrm{d}_{6}\right): \delta 4.01(\mathrm{~d}, 2 \mathrm{H}), 4,75(\mathrm{~s}, 2 \mathrm{H}), 5.15(\mathrm{t}, \mathrm{NH}), 6.55-7.40(\mathrm{~m}, 10 \mathrm{Ar}-$ $\mathrm{H}), 10.00(\mathrm{~s}, \mathrm{OH}) ; \mathrm{C}_{16} \mathrm{H}_{19} \mathrm{~N}_{2} \mathrm{O}_{2}$ (256.3).

\section{References}

1 (a) Coletti-Previero, M.-A.; Crastes de Paulet, A.; Matras, H; Previero (1982), A. Biochem. Biophys. Res. Commun.107: 465; (b) Smith, R.A.; Coles, P. J.; Spencer, R.W., Copp, L.J.; Jones, C. S.; Krantz (1988) A. Biochem. Biophys. Res. Commun. 155: 1201

2 (a) Michaelides, M.R; Curtin, M.L. (1999), Curr. Pharm. Design 5: 787; (b) Yamamoto, M; Ikeda, S.; Kondo, H.; Shintaro (2002), I. Bioorg. Med. Chem. Lett. 12: 375

3 Meier, C.; Boche (1990), G. Chem. Ber.123: 1691

4 (a) Meier, C.; Boche (1990), G. Chem. Ber. 123: 1699 (b) Meier, C.; Boche G. (1990), Tetrahedron Lett. 31: 1685

5 Ono, M; Itoh, I. (1989), Tetrahedron Lett. 30: 207

6. Exner, O; Simon, W. (1965), Collect. Czech. Chem. Commun. 30: 4078 